\section{Onfalocele gigante. Presentación de un caso en sesión anatomopatológica y consideraciones generales}

\author{
Gigant omphalocele. Anatomo- \\ clinical case presentation and general \\ considerations
}

María del Carmen Ávila-Iglesias ${ }^{1}$

Pedro Pasquel-García²

${ }^{1}$ Departamento de Neonatología.

2 Departamento de Patología.

Instituto Nacional de Pediatría, México.

\section{PRESENTACIÓN DEL CASO}

Paciente masculino producto de la primera gestación de madre de 20 años, originaria de medio rural en Guerrero. Control prenatal irregular en un Centro de Salud. Se efectuó un ultrasonido con aparente alteración del cordón umbilical no especificada, sin recibir manejo ni referencia a tercer nivel. Obtenido por vía abdominal por sufrimiento fetal agudo en clínica rural, presentó Ilanto inmediato, pesó 2800 g y se desconocen talla y Apgar al nacer. Un onfalocele gigante fue evidente desde el nacimiento y fue trasladado por sus propios familiares durante seis horas en malas condiciones, sin aporte enteral ni parenteral y con defecto abdominal sin cubrir. A su ingreso al Instituto Nacional de Pediatría se encontraba en estado de choque, por lo que se le intubó y se le pasaron 2 bolos de cristaloide a $10 \mathrm{~mL} / \mathrm{kg}$. Se describió un onfalocele gigante de $10 \mathrm{~cm}$ cubierto por gelatina de Wharton, con hígado, intestino delgado y estómago. Se practicó aseo con yodopovidona y se cubrió el defecto con gasa, sin posibilidad de colocar silo debido al gran tamaño del defecto y a la cavidad abdominal estrecha.

Se inició esquema antibiótico con ampicilina y amikacina. En los estudios de extensión se documentó cardiopatía congénita con comunicación interauricular de $4 \mathrm{~mm}$, insuficiencia tricuspídea leve y persistencia de conducto arterioso pequeño; se descartaron otras malformaciones asociadas. Al segundo día de vida se iniciaron curaciones con sulfadiacina de plata. Durante su
Recibido: 25 de febrero del 2015

Aceptado: 16 de abril del 2015

Correspondencia: María del Carmen Ávila Iglesias mcavilai@yahoo.com

Este artículo debe citarse como

Ávila-Iglesias MC, Pasquel-García P. Onfalocele gigante. Presentación de un caso en sesión anatomopatológica y consideraciones generales. Acta Pediatr Mex 2015;36:182-188. 
internamiento padeció neumonía asociada con ventilador que fue tratada con vancomicina, cefepima y posteriormente meropenem, así como sepsis por hongos tratada con anfotericina B. No fue posible incrementar vía oral a más de $80 \mathrm{~mL} /$ kg por lo que se utilizó alimentación mixta con fórmula de inicio y nutrición parenteral. Continuó con curaciones diarias con sulfadiacina de plata.

A los 45 días de vida el onfalocele estaba cubierto en $90 \%$ con una capa escarificada, costrosa, no epitelializada; fue evaluado por cirugía plástica que indicó cambio a curaciones con gasas envaselinadas y gel coloide con intención de colocar parche de colágeno o malla Epifast $^{\circledR}$. A los 60 días de vida el paciente tuvo crisis convulsivas y se inicio tratamiento con difenilhidantoína. A los 65 días de vida presentó súbitamente choque séptico sin germen aislado y paro cardiorrespiratorio irreversible a maniobras avanzadas de reanimación.

\section{COMENTARIO CLÍNICO}

Se trató de un paciente proveniente de un medio rural, sin diagnóstico prenatal certero y que no fue referido oportunamente a un tercer nivel de atención. Se define como onfalocele gigante aquel cuyo diámetro excede los $5 \mathrm{~cm}$ y contiene parte del hígado (más de $75 \%$ del hígado se encuentra en el saco). ${ }^{1}$ La incidencia reportada a escala mundial es de 1 por cada 5000 embarazos y 0.8 por cada 10000 recién nacidos vivos. Esta diferencia se debe a que con frecuencia el onfalocele se asocia con otras malformaciones y cariotipos anormales (40 y 50\%, respectivamente) lo cual está directamente relacionado con aumento en la pérdida fetal. ${ }^{2,3}$ No existen datos exactos sobre la incidencia de onfalocele en México; sin embargo, existen reportes de que el onfalocele es más frecuente en poblaciones pobres y remotas, analfabetas y con acceso limitado al adecuado manejo perinatal..$^{4,5}$
La falta de diagnóstico prenatal y de referencia oportuna a un tercer nivel de atención, así como un traslado inadecuado, están asociados con mal pronóstico. ${ }^{4,5}$ La sensibilidad para el diagnóstico del onfalocele por ultrasonido es de $75 \%$ y puede ser detectado a partir de las 18 semanas de gestación. Las características ultrasonográficas para establecer el diagnóstico son: la posición del defecto en relación con el cordón umbilical, el tamaño del defecto, los órganos eviscerados y la presencia de membranas. ${ }^{6}$ A pesar de contar con un ultrasonido obstétrico anormal, la madre del paciente no fue referida para corroborar o descartar la alteración. La resonancia magnética puede jugar un papel importante cuando el diagnóstico por ultrasonido es incierto y para evaluar malformaciones asociadas. ${ }^{7}$ Además del diagnóstico se tuvieron que haber buscado anomalías asociadas y determinar un cariotipo. La detección temprana hubiese permitido un asesoramiento adecuado a los padres y planear el manejo obstétrico. ${ }^{2,8}$

En el caso de onfaloceles aislados detectados en el primer trimestre la desproporción visceral-abdominal (siendo mayor el diámetro del onfalocele que el de la cavidad abdominal) y el contenido del onfalocele pueden predecir el pronóstico perinatal. ${ }^{9}$ La elevación de la alfafetoproteína sérica materna, asociada con un ultrasonido del segundo trimestre, es positiva para el diagnóstico de defectos de la pared abdominal en 98\% de los casos. ${ }^{1}$ Además de los estudios de imagen se pudo haber realizado amniocentesis en la búsqueda de elevación de alfafetoproteína y acetilcolinesterasa. En las gastrosquisis la alfafetoproteína está invariablemente elevada, mientras que en el onfalocele es menos confiable. En el segundo trimestre la cantidad de alfafetoproteína es 9.42 veces el valor normal para la gastrosquisis y 4.19 veces el valor normal para onfalocele. La acetilcolinesterasa se eleva en $80 \%$ de las gastrosquisis y en $27 \%$ de los pacientes con onfalocele. ${ }^{10}$ 
La atención perinatal y el traslado por familiares sin ningún tipo de tratamiento de soporte condicionaron que el paciente llegara en estado de choque, impactando directamente en su pronóstico. Idealmente se debió haber referido a un tercer nivel para su atención perinatal. La realización de pruebas sin estrés desde las 32 semanas de gestación y la evaluación del crecimiento intrauterino, por ultrasonido de rutina durante todo el tercer trimestre, hubieran permitido evaluar las condiciones generales del feto en las últimas etapas del embarazo. ${ }^{1}$ No existe una recomendación basada en la evidencia que determine la vía de nacimiento de elección en el caso de onfalocele gigante pero la mayoría de los autores recomienda el nacimiento por cesárea electiva, en especial en los casos de herniación del hígado debido al riesgo de rotura y sangrado durante el parto vaginal. ${ }^{11,12}$ Se debe prolongar el embarazo lo más cercano al término posible.

El manejo inmediato del paciente debió incluir protección de la vía aérea con intubación y ventilación mecánica si fuese necesario; reanimación con líquidos (ya que el onfalocele predispone a una mayor pérdida de líquidos insensibles, aunque no tan drásticamente como la gastrosquisis), descompresión gástrica, protección del saco con apósitos remojados en solución salina. Después de la estabilización inicial el paciente debió ser trasladado en ambulancia con los cuidados apropiados (i.e. temperatura, líquidos intravenosos, aporte de glucosa, vigilancia estrecha de signos vitales y acompañado por un médico). ${ }^{13}$

Una vez que el paciente llegó a nuestra institución los esfuerzos se dirigieron a estabilizarlo y estudiar malformaciones asociadas. Se identificó malformación única en el corazón con cardiopatía congénita de flujo pulmonar aumentado (comunicación interauricular de $4 \mathrm{~mm}$, insuficiencia tricuspídea leve y persistencia del conducto arterioso pequeño).
El manejo definitivo del onfalocele depende tanto del tamaño del defecto como de la integridad del saco (intacto o perforado). Dependiendo del momento del cierre el manejo se clasifica en: cierre primario, por etapas y tardío.

Debido al tamaño del onfalocele las opciones terapéuticas se limitaron a intentar un cierre tardío que se define como la epitelialización del saco amniótico intacto o escisión de la bolsa con el cierre posterior de la piel que lo recubre. La reparación del defecto fascial remanente puede realizarse incluso después de la infancia. Varios métodos han sido empleados para la epitelialización: mercurocromo, yodopovidona, sulfadiacina de plata y neomicina-bacitracina. El mercurocromo ya no se utiliza debido a que se le asocia con intoxicación por mercurio.

La yodopovidona y la sulfadiacina de plata se han convertido en los agentes esclerosantes de elección. La sulfadiacina de plata ha demostrado ser segura y no tener efectos secundarios; sin embargo su aplicación y retiro pueden causar sangrado del tejido de granulación subyacente y es más difícil para los padres aplicarla en el hogar. No obstante, los resultados en general son excelentes y con pocas complicaciones. ${ }^{14} \mathrm{La}$ yodopovidona es de fácil administración para los padres pero se han informado casos en los que la absorción del yodo se asoció con hipotiroidismo clínico. La asociación de yodopovidona con antibiótico en polvo promueve la epitelialización rápida y disminuye el riesgo de hipotiroidismo. ${ }^{15}$

Otro tratamiento conservador es la aplicación de eosina disódica acuosa a $2 \%$, que ha demostrado ser simple de aplicar y tener un costo bajo. La madre puede aprender fácilmente como aplicarla en casa reduciendo la duración de la estancia hospitalaria. ${ }^{16}$ El manejo con cierre tardío tiene la ventaja general de evitar por completo la cirugía abdominal en el período neonatal, lo que también disminuye los riesgos asociados de cierre 
abdominal apretado en una cavidad estrecha, así como las complicaciones observadas en el cierre por etapas. El manejo conservador disminuye el tiempo de inicio de la alimentación enteral, así como la sepsis y la estancia hospitalaria. ${ }^{17}$

A pesar de no tener malformaciones importantes asociadas, lo que se hubiese asociado con un mejor pronóstico de supervivencia, la falta de diagnóstico prenatal, el nacimiento en una clínica rural y las malas condiciones de traslado determinaron la morbilidad y mal pronóstico del paciente a su ingreso. Las complicaciones asociadas con la estancia intrahospitalaria prolongada fueron condicionantes para la mala evolución y defunción.

\section{HALLAZGOS ANATOMOPATOLÓGICOS}

El onfalocele midió $10 \times 8 \times 7 \mathrm{~cm}$, se encontraba íntegro y la membrana estaba muy engrosada con esclerosis y momificación (Figura 1). El saco contenía parte del hígado, estómago e intestino delgado. Se documentaron complicaciones producto de una hospitalización prolongada: en primer lugar una tromboflebitis de la vena cava superior con secuelas embólicas en los pulmones en donde, además, había cambios de daño

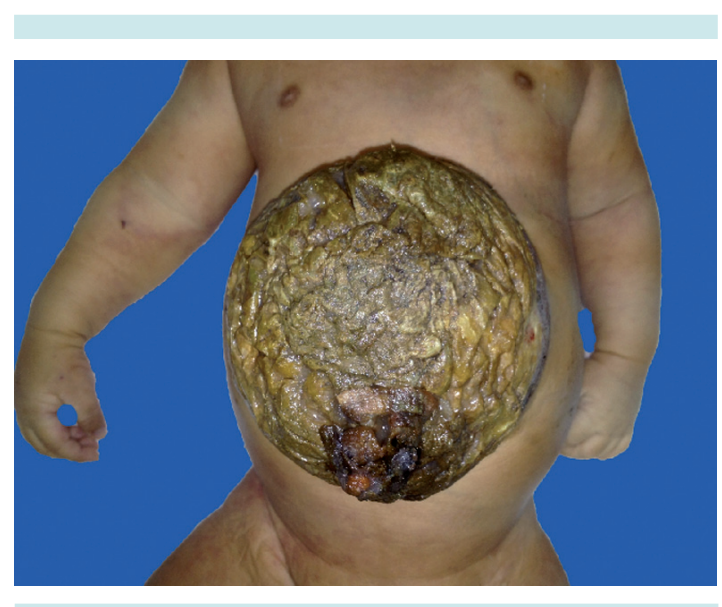

Figura 1. Onfalocele visto en su cara anterior. alveolar difuso crónico (Figura 2). Se encontraron datos anatómicos de sepsis en el hígado (Figura 3) y en el bazo (Figura 4). La bacteriología post mortem reportó Klebsiella pneumoniae y Enterococcus faecalis en pulmón, bazo, hígado y líquido cefalorraquídeo.

\section{DISCUSIÓN}

De acuerdo con los hallazgos anatomopatológicos las complicaciones infecciosas y trombóticas

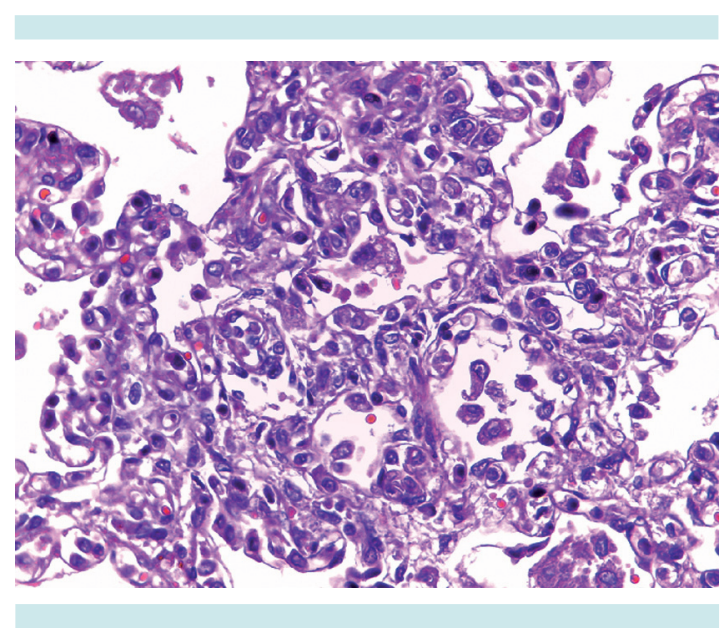

Figura 2. Parénquima pulmonar: tabiques alveolares engrosados con depósito de colágena (40x).

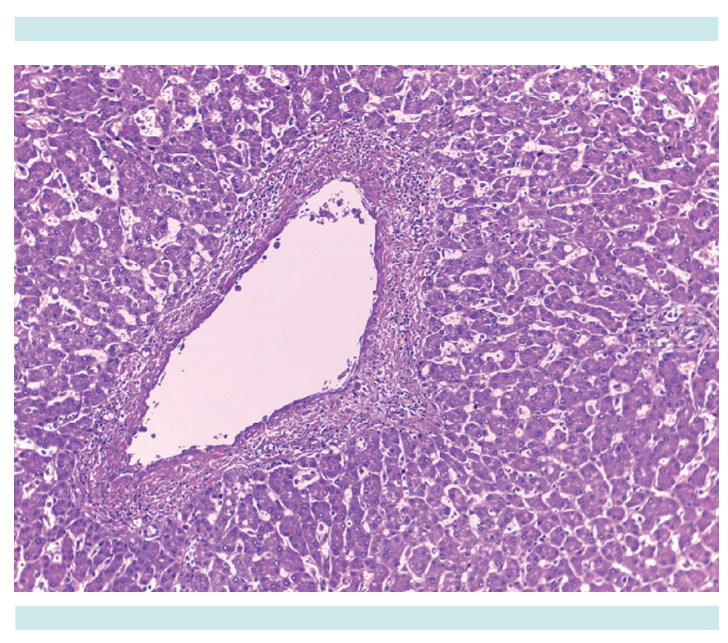

Figura 3. Parénquima hepático: flebitis de suprahepáticas (10x). 


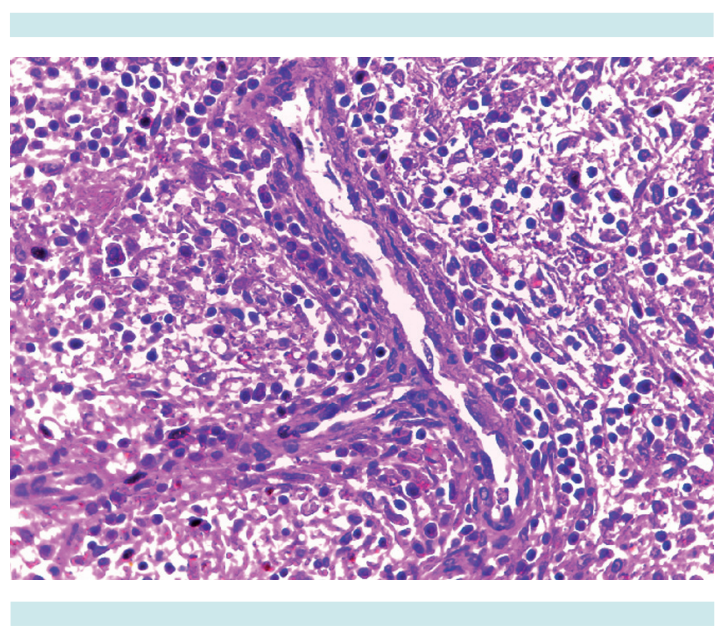

Figura 4. Flebitis trabecular en bazo.

fueron las condicionantes de la muerte del paciente. La imposibilidad de completar el aporte nutricional por vía enteral y depender de un acceso venoso por tiempo prolongado favoreció, en parte, las dos complicaciones.

En este sentido es importante contrastar la experiencia publicada por algunos centros que sirven como referencia y modelo a seguir. En el 2002 el grupo del Hospital de Especialidades del Centro Médico Nacional Noroeste publicó una serie de casos de 15 pacientes con malformaciones de la pared abdominal (11 gastrosquisis y 4 onfaloceles) diagnosticados prenatalmente y a quienes se les ofreció cirugía neonatal inmediata. Sus pacientes incluyeron binomios que vivían en otras regiones, oportunamente diagnosticados y referidos a su centro en el mejor medio de transporte (el útero gestante). Sus resultados más notables fueron ausencia de sepsis y cierre primario en 4 y 3 pacientes, respectivamente. ${ }^{18}$

Por otro lado, en el Instituto Nacional de Perinatología Isidro Espinosa de los Reyes durante 10 años (1998-2008) se atendieron un total de 198 pacientes con defectos de pared abdominal (152 con gastrosquisis, 41 con onfalocele, 4 con pentalogía de Cantrell y 1 con hernia de cordón); en 110 pacientes se colocó un silo, en 81 se logró cierre primario y en 7 no se realizó ningún tipo de cirugía. En 127 pacientes se realizó la intervención quirúrgica entre 1 y 3 días; en 40 se realizó en las primeras 24 horas; en 17 entre los 4 y 6 días; en 7 pacientes entre los 7 y 9 días y 2 pacientes en 10 días o más. En esa serie 172 pacientes sobrevivieron $(86.8 \%){ }^{19}$

Ambos ejemplos reflejan que la atención ideal de estos pacientes debe incluir el diagnóstico prenatal y la referencia oportuna a un centro especializado con la experiencia para el tratamiento definitivo.

El hecho de que durante el embarazo de este caso se reportó un ultrasonido con una alteración del cordón umbilical al que no se le dio seguimiento es también una llamada de atención y un punto para reflexionar. La ultrasonografía obstétrica rutinaria es controvertida; el estudio RADIUS mostró que la ultrasonografía obstétrica de rutina, comparada con la solicitada por indicación clínica, no modifica los desenlaces fetales ni maternos en cuanto a supervivencia. Sin embargo, sí se observó una tendencia a mayor supervivencia en neonatos con condiciones graves (75 vs. 52\%), menor uso de agentes tocolíticos y menor tasa de embarazos postérmino. Así mismo, la ultrasonografía obstétrica identificó un mayor número de anormalidades fetales antes de las 24 semanas de gestación (17 vs. 5\%). ${ }^{20}$

Recientemente, la Colaboración Cochrane publicó una revisión sistemática y metanálisis que comparaban los beneficios de la ultrasonografía obstétrica rutinaria con la solicitado bajo una indicación. Sus resultados evidencian los beneficios del ultrasonido rutinario disminuyendo la falla de detección de embarazos múltiples (RR 0.07 IC 95\% 0.03-0.17) de la inducción de trabajo de parto por embarazos "postérmino" (RR 0.59 IC 95\% 0.42-0.83). ${ }^{21}$ 
En el 2002 se publicó una revisión sistemática de los estudios que analizan el costo-efectividad del ultrasonido de rutina y se evidenciaron dos puntos importantes: las habilidades del ultrasonografista y el tiempo en el que se realiza el estudio son determinantes en el costo-efectividad de cualquier programa de ultrasonografía obstétrica. Por otro lado, parece ser que el costo-efectividad se mantiene únicamente si la terminación del embarazo es aceptable.22

En nuestro país no tenemos ningún estudio similar que permita conocer si estos hallazgos son reproducibles y recomendables para nuestra población. Es un campo de oportunidad que requiere atención dado que, muy probablemente, podrían disminuirse costos del uso de servicios de salud y mejorar nuestras prácticas clínicas hacia esta población vulnerable.

Existen lineamientos internacionales para la adecuada reanimación al nacimiento y para el correcto traslado de pacientes neonatos que optimizan la supervivencia. A escala mundial se deben seguir, de manera estandarizada, los lineamientos del Programa de Reanimación Neonatal $6^{\text {a }}$ edición (Neonatal Resuscitation Program o NRP) para la atención en cada nacimiento en un medio hospitalario. Existe también un programa de la Academia Americana de Pediatría llamado "Ayudando a los bebés a respirar" (Helping babies breathe. The Golden Minute o HBB) enfocado a capacitar al personal para atender nacimientos en escenarios con recursos limitados. El programa STABLE (SugarTemperature-Airway-Blood pressure-Lab work and Emotional support program) tiene como objetivos específicos la estabilización y el manejo adecuado del neonato antes y durante el traslado. El programa ACORN (Acute Care of at Risk Newborns) está enfocado en la evaluación y manejo del neonato en estado crítico. Existe también el Course on Neonatal and Pediatric Critical Care Transport Medicine impartido por la
Sección de Medicina de Traslado de la Academia Americana de Pediatría. ${ }^{23}$ Si bien no es factible capacitar a todos los médicos que en su quehacer profesional atienden a pacientes pediátricos, sí es importante recalcar que el sentido común y la ética profesional por ningún motivo deben permitir que un paciente de estas características sea enviado por sus propios medios.

La atención de neonatos con malformaciones congénitas es compleja y requiere la mayoría de las veces de atención especializada. El mejor transporte que podemos ofrecerle a un neonato con malformación congénita es el útero de su madre y la única forma de ofrecer esta opción es con un diagnóstico prenatal oportuno. Es nuestro deber como profesionales de la salud fomentar estas posibilidades para mejorar la salud de nuestra población pediátrica.

\section{REFERENCIAS}

1. Davis A. Challenges of Giant Omphalocele: From Fetal Diagnosis to Follow-up. Neo Reviews 2008;9:338-374.

2. Cohen-Overbeek TE. Omphalocele: comparison of outcome following prenatal or postnatal diagnosis. Ultrasound Obstet Gynecol 2010;36:687-692.

3. Brantberg A. Characteristics and outcome of 90 cases of fetal omphalocele. Ultrasound Obstet Gynecol 2005;26:527-537.

4. Uba FA, Chirdan LB. Omphalocele and gastroschisis: management in a developing country. Nig J Surg Res 2003;5:57-61.

5. Osifo OD, et al. Omphalocele management using goaloriented classification in African centre with limited resources.J Trop Pediatr 2011;57(4):286-8.

6. Emanuel PG, et al. Prenatal detection of anterior abdominal wall defects with US. Radiographics 1995;15:517-530.

7. Breysem $L$, et al. The value of fast $M R$ imaging as an adjunct to ultrasound in prenatal diagnosis. Eur Radiol 2003;13:1538-1548.

8. Dykes EH. Prenatal diagnosis and management of abdominal wall defects. Semin Pediatr Surg 1996;5:90-94.

9. Tassin $\mathrm{M}$, et al. Omphalocele in the first trimester: prediction of perinatal outcome. Prenatal Diagnosis 2013;33:497501.

10. Mortellaro V, et al. Review of the evidence on the closure of abdominal wall defects. Pediatr Surg Int 2011;27:391-397. 
11. Wilson RD, Johnson MP. Congenital abdominal wall defects: an update. Fetal Diagn Ther 2004;19:385-398.

12. Lurie S, Sherman D, Bukovsky I. Omphalocele delivery enigma: the best mode of delivery still remains dubious. Eur J Obstet Gynecol Reprod Biol 1999;82:19-22.

13. Katensberg ZJ, Dutta S. Ventral abdominal wall defects. Neo Reviews 2013;14(8):402-11.

14. Ein SH, Langer JC. Delayed management of giant omphalocele using silver sulfadiazine cream: an 18-year experience. J Pediatr Surg 2012;47(3):494-500.

15. Pandey $\mathrm{V}$, et al. Non-operative management of giant omphalocele with topical povidone-iodine and powdered antibiotic combination: early experience from a tertiary centre. Pediatr Surg Int 2014;30(4):407-11.

16. Kouame BD, et al. Outcomes of conservative treatment of giant omphaloceles with dissodic $2 \%$ aqueous eosin: 15 years' experience. Afr J Paediatr Surg 2014;11(2):170-3.

17. Sönmez K, et al. A strategy for treatment of giant omphalocele. World J Pediatr 2010;6(3):274-7.

18. Gómez-Alcalá AV, Jiménez-Muñoz J, Rodríguez-Rodríguez A, Ríos-Félix A, Espino-Valle l et al. Cirugía neonatal inmediata: experiencia inicial en el tratamiento de gastrosquisis y onfalocele en el noroeste de México. Gac Med Mex 2002;138(6):511-17.
19. García-Cervantes L, Guido-Ramíres O \& Fernández-Carrocera L. Epidemiología de los defectos de pared abdominal en el Instituto Nacional de Perinatología Isidro Espinosa de los Reyes en el último decenio. Tesis de Subespecialidad, Universidad Nacional Autónoma de México, 2009.

20. Crane JP, LeFevre ML, Winborn RC, Evans JK, Ewigman G, Bain RP, et al. A randomized trial of prenatal ultrasonographic screening: impact on the detection, management, and outcome of anomalous fetuses. American Journal of Obstetrics and Gynecology 1994;171:392-9.

21. Whitworth M, Bricker L, Neilson J, Dowswell T. Ultrasound for fetal assessment in early pregnancy. Cochrane Database Syst Rev: CD007058. doi:10.1002/14651858.CD007058. pub2

22. Roberts T, Henderson J, Mugford M, Bricker L, Neilson J, Garcia J. Antenatal ultrasound screening for fetal abnormalities: a systematic review of studies of cost and cost effectiveness. Br J Obstet Gynaecol 109:44-56.

23. Stroud MH., Trautman MS., Meyer K., Moss MM., Schwartz $\mathrm{HP}$, et al. Pediatric and Neonatal Interfacility Transport: Results From a National Consensus Conference. Pediatrics 2013;132(2):1-8

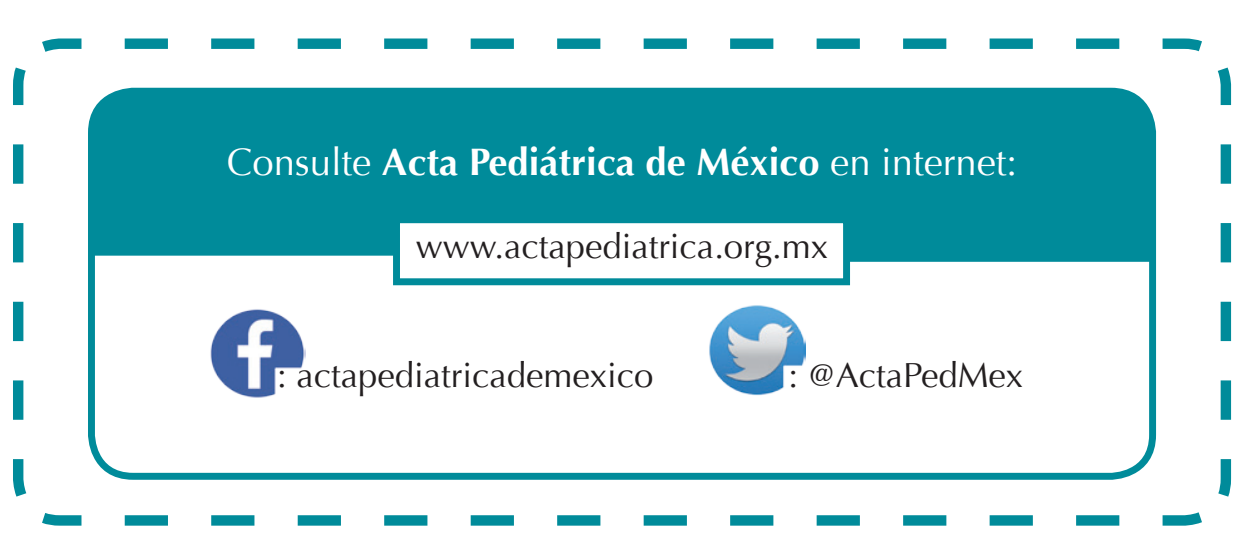

